\title{
IkasSasoi: movilizando la comunidad para fomentar la actividad física de lapoblación adolescente de Irun IkasSasoi: mobilizing the community to promotephysical activity among teenagers in Irun
}

Iñaki Iturrioz*, Iñaki Arrese-Igor*, David Izaga*, Jokin Vesga*, Pedro Iturrioz**, Álvaro Cano***, Miguel Á. Izquierdo****, José Ramon Otegi**** y Arantxa Uranga*****

*Ayuntamiento de Irun, **Unidad Docente de Medicina Familiar y Comunitaria. Gobierno Vasco, ***Kabia Heziketa, SL. Coordinador del Programa de Educación en Medio Abierto, ****Gobierno Vasco. Profesor de Educación Física, *****Subdirección Territorial de Salud Pública de Gipuzkoa. Gobierno Vasco

Resumen. La adolescencia es un período de especial interés para el fomento de hábitos saludables, entre ellos el de practicar actividad física con regularidad. Diversas intervenciones que han actuado sobre este grupo de población, se han centrado tradicionalmente en el ámbito del centro escolar. El conocimiento aportado por las investigaciones en el área del fomento de la actividad física parece señalar a las estrategias multicomponente como las más indicadas. IkasSasoi es una iniciativa multicomponente promovida por el Ayuntamiento de Irun en la que participan distintas áreas municipales y distintos agentes de la comunidad que persigue fomentar la actividad física y la evitación de la conducta sedentaria entre los y las escolares que cursan E.S.O. en Irun. IkasSasoi contempla un diagnóstico inicial, para lo cual se ha administrado un cuestionario sobre hábitos de actividad física a 1.797 sujetos y se han desarrollado cuatro grupos focales. A partir de la información recabada, el grupo de trabajo ha aprobado llevar a cabo 23 intervenciones, de las cuales están en marcha o ya ejecutadas 19. IkasSasoi, ha permitido conocer en profundidad los hábitos y percepciones de las y los jóvenes y ha generado una red intersectorial consolidada. El programa está ubicando a la población adolescente en la agenda de las políticas locales vinculadas con la actividad física. Los principales aspectos a mejorar se refieren al incumplimiento de los plazos de intervención en acciones concretas y a la desigual implicación de los agentes, especialmente en los centros educativos.

Palabras clave. actividad física, fomento, adolescentes, intervención, comunitario, IkasSasoi.

Abstract. The adolescence is a period of particular interest to promote healthy habits, including regular physical activity. Interventions addressed to this population group have traditionally focused on the area of the school. The knowledge obtained from research in the area of promoting physical activity seems to draw multicomponent strategies as the most appropriate. IkasSasoi is a multicomponent initiative promoted by the City Council of Irun in which various municipal departments and community agents take part and which's aim is to promote physical activity and avoidance of sedentary behavior among adolescents studying ESO in Irun. IkasSasoi includes an initial diagnosis, for which it has been administered a questionnaire on physical activity habits to 1.797 subjects and developed four focus groups. From the information gathered, the working group has validated 23 interventions, 19 of them are underway or already completed. IkasSasoi, has revealed the habits and perceptions of the teenagers and generated a consolidated intersectorial network. The program is placing adolescents on the agenda of local policies related to physical activity. The main areas for improvement relate to missed intervention's deadlines of interventions and the unequal involvement of actors, especially in schools.

Keywords. physical activity, promotion, adolescents, intervention, community, IkasSasoi.

\section{Introducción}

La actividad física juega un papel fundamental en la salud y el bienestar de los y las adolescentes. Contribuye a su desarrollo físico, social, emocional y psicológico (Kelly, Matthews \& Foster, 2012; Biddle \&Asare, 2011; Janssen \& Leblanc, 2010) y, a través del deporte, puede aportar valores positivos en su educación (Singh, Uijtdewilligen, Twisk, van Mechelen, Fedewa, y Ahn, 2011; Mulholland, 2008). La Organización Mundial de la Salud ha establecido los mínimos recomendados de actividad física que la población adolescente debería alcanzar para prevenir la enfermedad. Las investigaciones llevadas a cabo en este ámbito parecen indicar que la práctica de actividad física moderada o vigorosa durante un mínimo de 60 minutos diarios ayuda a la infancia y juventud a mantener un perfil de riesgo cardiorrespiratorio y metabólico saludable (OMS, 2010).

Determinados condicionantes conducen a establecer la adolescencia como un período de especial relevancia en la acción de fomento de la actividad física dirigida a la población. De un lado parece observarse que los hábitos que se instalan en la adolescencia tales como hacer actividad física, tomar frutas o verduras, consumir alcohol o fumar suelen persistir durante mucho tiempo. Estos hábitos suelen ser, además, de difícil erradicación en la edad adulta(OMS, 2007; Telama, Yang, Viikari, Wanne y Raitakari, 2005). Por otra parte, en la adolescencia se observa una significativa tasa de abandono de la práctica deportiva (Currie et al., 2008), en concreto, en las edades asociadas al cambio de ciclo educativo se observa un importante decremento en los niveles de actividad, especialmente entre la chicas (CSD, 2011).

Estudios recientes llevados a cabo en el Estado y que miden la actividad física en la población adolescente a través de instrumentos de

Fecha recepción: 30-09-14- Fecha envío revisores: 30-09-14- Fecha de aceptación: 15-11-14 Iñaki Iturrioz Rosell iiturrioz@irun.org medición objetiva, han detectado que una amplia mayoría de la población adolescente incumple los niveles mínimos recomendados por la OMS (Abarca-Sos, Zaragoza, Generelo y Julián, 2010; Oviedo et al, 2013). El estudio «Análisis de la actividad física en escolares de medio urbano» (Hernández, Ferrando, Quilez, Aragonés y Terreros, 2008), refleja las conclusiones de una investigación llevada a cabo con 85 sujetos de entre 10 y 14 años en la ciudad de Zaragoza empleando instrumentos de medición objetiva:

«Encontramos diferentes patrones de actividad física en función de la edad y del sexo, y describimos que estas diferencias se deben principalmente a la práctica de deportes organizados. /. . . El tiempo libre de estos escolares es muy sedentario y dedican la mayor parte del tiempo a actividades de bajo gasto energético. Hay una gran dependencia del transporte motorizado y se invierte demasiado tiempo en actividades de tipo académico y muy poco en actividad física. Estos escolares presentan un alto índice de inactividad en comparación con poblaciones de otros países y no alcanza los índices mínimos establecidos para las patologías ligadas al sedentarismo. Por ello definimos el estado de la cuestión como altamente preocupante».

\section{La promoción de la actividad física y el deporte desde el Ayunta-} miento de Irun

El Ayuntamiento de Irun ha adoptado un cambio en el modelo de gestión del deporte en la ciudad, que implica la integración del extinto Patronato de Deportes en el Ayuntamiento y la gestión indirecta de los dos grandes equipamientos de la ciudad (Ayuntamiento de Irun, 2012), abriendo la puerta a una oportunidad para reorientar la misión de la estructura municipal de atención al Deporte pasando de un modelo centrado en la gestión de los recursos a un modelo más integral y con una perspectiva más amplia, representada en la figura 1.

El Servicio Municipal de Deportes acentúa así su vertiente como facilitador de la actividad física en el municipio, valiéndose de todos los recursos existentes en el municipio y orientado a toda la población, población activa y población inactiva. El papel hasta ahora preponde- 

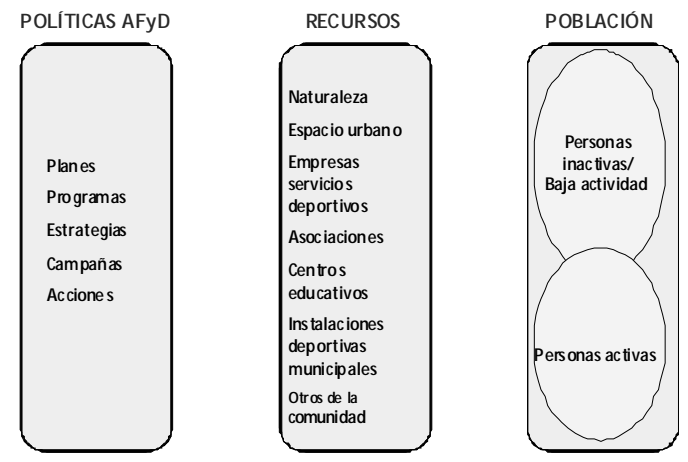

Figura 1. Marco referencial de la orientación facil itadora de la acción municipal en el fomento de la activida

Fuente: Iturrioz, 2012a

rante como oferente de servicios deportivos tiene lugar pero en el nivel de los recursos, situándose el Ayuntamiento en una posición más abierta y dinámica respecto de la elaboración de políticas, programas y acciones orientados a la promoción de la actividad física.

A este nivel, para resultar efectivos deben tenerse en cuenta los determinantes de la actividad física (factores que influyen en que las personas sean activas o no) y las estrategias que la evidencia científica señala como más efectivas en el fomento de la actividad física. Uno de los grandes fundamentos que respaldan la efectividad en el fomento de la actividad física es el de soportar las acciones en intervenciones multicomponente transversales (OMS, 2009; GAPA, 2011; Bellew, Bauman, Martin, Bull \& Matsudo, 2011), intervenciones que incluyen distintos programas coordinados entre sí, que actúan a distintos niveles y que involucran a distintas áreas: promoción de la salud, urbanismo, educación, igualdad, movilidad, medio ambiente. .

Conviene, así, habilitar una iniciativa comunitaria, un funcionamiento más colaborativo con otras áreas municipales y otros agentes de la comunidad. Incluir en su campo de acción un concepto de actividad física amplio, que incluya los desplazamientos activos, la actividad física ocupacional, la actividad física en el tiempo libre, el propio deporte como vertiente más reglamentada y orientada al resultado. Considerando incluso la evitación de la conducta sedentaria (permanecer sentada mucho tiempo, ver la televisión durante horas...) como variable independiente por sus implicaciones en relación con los beneficios de la actividad física regular (Brown, Bauman \& Owen, 2009; Hamilton, Hamilton \& Zderic, 2007).

\section{Estrategias prometedoras para el fomento de la actividad física en} la población adolescente

Una revisión sobre programas escolares de actividad física para promover la actividad física y el buen estado físico en niños y adolescentes encuentra pruebas convincentes de que las intervenciones tienen una repercusión positiva sobre cuatro de las nueve medidas de resultado estudiadas: tiempo dedicado a la actividad física, tiempo dedicado a ver la televisión, consumo máximo de oxígeno y colesterol en sangre. De cara a la intervención, se observó que, como mínimo, una combinación de materiales educativos impresos y cambios orientados a promover la actividad física en el programa escolar de estudios produjeron efectos positivos (Dobbins, Husson, DeCorby \& LaRocca, 2013).

Una revisión científica sobre intervenciones basadas en la escuela (Kriemler et al., 2011), encuentra las estrategias de intervención multicomponente las más consistentes, observando una mayor efectividad en las que incorporan a las familias.

Otra revisión sistemática efectuada sobre estudios con adolescentes europeos (Crutzen, 2010) alcanza diversas conclusiones: las intervenciones basadas en la escuela conllevan generalmente un incremento en los niveles de actividad física, el nivel de actividad física se incrementaba con el apoyo de los pares y la influencia de cambios en el entorno físico próximo. Si una intervención buscaba intervenir sobre otros hábitos además de la actividad física, la intervención resultaba menos efectiva en incremento de actividad física.

Un análisis de la evidencia de efectividad en promoción de la actividad física (van Sluijs, McMinn \& Griffin, 2007), encuentra fuerte evidencia en las estrategias multicomponente y en las desarrolladas en el marco del centro educativo con apoyo de la comunidad o de las familias.

Una revisión acerca de intervenciones efectivas en fomento de la actividad física en adolescentes (Murillo et al., 2013) conduce a los autores a proponer cinco líneas de acción en intervenciones basadas en la escuela: diseño de intervenciones multicomponente que impulsen el empoderamiento de los miembros de la comunidad escolar, desarrollo de mejoras en el currículo escolar de Educación Física, diseñar e implementar programas no curriculares y actividades para promover la actividad física, incluir intervenciones apoyadas en la informática y diseñar intervenciones específicas dirigidas a los intereses de las chicas.

Un proyecto desarrollado para detectar los factores más relevantes en los que debieran incidir las acciones de fomento de la actividad física en jóvenes (Kelly et al., 2012), propone tres áreas esenciales:

- El entorno físico: ubicación (asegurar facilidad de acceso), costes (mantenerlos bajos), incluir si es posible actividades al aire libre, evitar zonas con contaminación, caminar y bicicleta, equipamientos e instalaciones deportivas (asegurando la calidad), clubes deportivos (facilitar acceso a centros de los clubes como lugar de encuentro dejóvenes).

- El entorno social: cultura de la actividad (promover actitudes favorables), competición saludable (centrada en la mejora personal), incluir mentores, socialización (dotar de oportunidades para desarrollar amistades), sensibilización sobre la salud (enfatizando los beneficios), posibilidad de elección (incluir oportunidades), actividades de ámbito nacional y con la participación de personas famosas.

• La experiencia de la participación: independencia y autoeficacia, relajación como un resultado más de la actividad, evitar demasiada actividad y el riesgo de lesiones, diversión (asegurar que las actividades son divertidas).

De la síntesis del conocimiento trasladado por las investigaciones anteriormente expuestas, cabe observar una intervención centrada en el ámbito del centro educativo a la hora de abordar el fomento de la actividad física en la población adolescente. Sin duda el hecho de la enseñanza obligatoria hasta los 16 años ofrece la ventaja y oportunidad de encontrar a la totalidad de los y las adolescentes en el escenario del centro. Sin embargo, parece poco explorada una intervención dirigida a este grupo de población con una perspectiva más amplia, con perspectiva comunitaria, que involucre a todos los agentes de la comunidad, incluidos los correspondientes al ámbito propiamente educativo.

\section{Proyecto IkasSaso}

IkasSasoi es una iniciativa multicomponente promovida por el Ayuntamiento de Irun en la que participan distintas áreas municipales y distintos agentes de la comunidad que persigue fomentar la actividad física y la evitación de la conducta sedentaria entre los y las escolares que cursan E.S.O. en Irun.

En los cursos académicos 2003-04 y 2004-05, el Ayuntamiento de Irun, en colaboración con los centros educativos de Enseñanza Secundaria Obligatoria (E.S.O.) de la ciudad, llevaron a cabo un programa para el fomento de la actividad física entre los y las adolescentes que cursaban E.S.O. en el municipio. Básicamente el programa consistía en un diagnóstico previo de hábitos saludables, una intervención basada en las conclusiones del diagnóstico y una evaluación posterior. En el diagnóstico y posterior evaluación, se emplearon mediciones cuantitativas, materializadas a través de una encuesta, y mediciones cualitativas, a través de grupos focales de discusión. Antes de la intervención el 26,9\% de los chicos y el 53,2\% de las chicas podía calificarse como personas sedentarias. Después de la intervención, el 24,8\% de los chicos y el $51,0 \%$ de las chicas. Las chicas son considerablemente menos activas que los chicos. Las principales dificultades observadas por los jóvenes para hacer actividad física fueron, en orden descendente: no tener tiem- 
po, no gustarles hacer actividad física solas y resultar estresante. Uno de cada tres jóvenes mantenía hábitos de tiempo libre sedentarios (ver la televisión, jugar con ordenadores, videoconsolas...) en cuatro horas o más al día. El momento clave en cuanto al abandono de hábitos de actividad física se daba entre los 13 y los 14 años.

Durante el curso 2012-2013, el Ayuntamiento de Irun pone en marcha una nueva iniciativa para el fomento de la actividad física entre los y las adolescentes del municipio. La iniciativa se aborda con la perspectiva del Modelo Vasco de Actividad Física (Iturrioz, 2012b) y tiene en cuenta el punto de partida desde un grupo de población específico detectado como población objetivo -en este caso la adolescenciay se traducirá a acciones a llevar a cabo en distintos escenarios (no exclusivamente en el del centro educativo), atendiendo a los factores de índole individual, social y del entorno físico que influencian el comportamiento activo de chicos y chicas. Se trata, por lo tanto de una iniciativa comunitaria no enfocada exclusivamente al ámbito escolar, un programa orientado a la intervención que pretende hacer presente el apoyo de la comunidad para incrementar la actividad física de la población escolar que cursa E.S.O. en Irun.

El abordaje tiene además una perspectiva intersectorial que se refleja en la propia participación municipal, incorporando la dedicación de distintas áreas municipales al desarrollo del programa: Educación, Juventud, Deportes, Intervención Comunitaria, Igualdad, Movilidad, Seguridad Ciudadana y Euskera.

El grupo de trabajo conformado para el diseño y seguimiento del plan se compone de los siguientes integrantes: técnicos municipales de Educación, Juventud, Deportes, Movilidad y Euskera, técnico de Salud Pública del Gobierno Vasco, personal directivo de la Organización de Salud Integrada de la comarca Bidasoa del Sistema Vasco de SaludOsakidetza, miembro de Osatzen-Sociedad Vasca de Medicina de Familia y Comunitaria, técnicos de las empresas Kabia responsables de programas municipales de Juventud y Bienestar Social, personal docente de Educación Física de los 7 centros de Enseñanza Secundaria Obligatoria de Irun.

\section{Objetivos}

1. Incrementar el porcentaje de la población escolar de E.S.O. que alcanza los niveles mínimos de actividad física recomendados para este grupo de población (Iturrioz et al., 2012c).

2. Incrementar el porcentaje de personas que refieren una actitud favorable hacia la actividad física.

3. Reducir la percepción de barreras para el acceso a dicha práctica en el municipio.

4. Reducir la desigualdad proveniente de la incidencia en la población de los determinantes sociales de la práctica de actividad física y el mantenimiento de la conducta sedentaria.

5. Reducir el tiempo que durante el día la población dedica a actividades sedentarias y el permanecer de forma prolongada en actividades sedentarias como estar sentado, exposición ante pantallas de televisión, ordenador, etc.

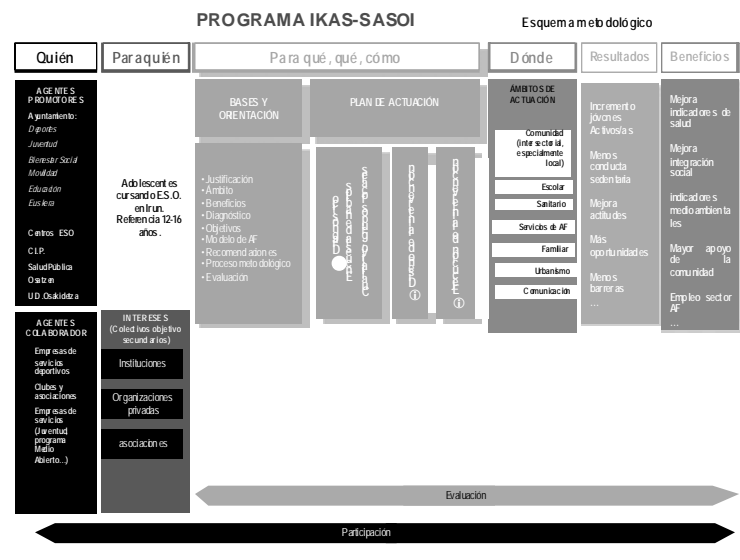

Figura 2. Modelo metodoló gico del programa IkasS asoi. Fuente: elaboración propia basado en Iturrioz et al.,2011.
Se considera, además, que de la consecución de los objetivos planteados se derivarán una serie de beneficios para la comunidad: mejora de la salud, de la integración social, del medio ambiente, de las redes sociales y del sentido de comunidad. El incremento de la actividad física ha evidenciado influencia directa en la consecución de gran parte de estos beneficios (Iturrioz et al, 2011).

\section{Metodología}

La metodología para el desarrollo del programa IkasSasoi se basa en un modelo lógico de desarrollo, inspirado en modelos propuestos en guías y programas para la promoción de la actividad física (Huhman, Heitzler \& Wong, 2004; US Department of Health and Human Services, 2002). Con este planteamiento se pretende asociar desde un inicio los objetivos e iniciativas desarrolladas en el marco del plan con los resultados y beneficios esperados.

En este apartado se refleja el esquema lógico planteado para el desarrollo de IkasSasoi (Figura 2), y que provee de una metodología de trabajo a los y las profesionales y actores de la actividad física que intervengan en su elaboración. También se han tomado en cuenta aspectos básicos en la metodología de procesos de democracia participativa, en especial en lo relativo a explicitar agentes de promoción, personas destinatarias y finalidad de la iniciativa (Villasante, 2010).

El primer apartado, trata de responder a la pregunta ¿quién?, ¿quién realiza las propuestas? Se define quién va a realizar las propuestas a la población adolescente. En este sentido, se ha destacado la importancia de una fuerte implicación a nivel comunitario en el que se comprometan administraciones, sociedad civil y ciudadanía. Se han establecido dos niveles de compromiso: promotor y dinamizador. Dentro de la Administración, se ha desvelado importante conseguir una fuerte implicación política (OMS, 2007).

En segundo lugar se expone a quién está dirigido IkasSasoi, para quiénes tiene que reportar un beneficio. IkasSasoi está dirigido a la población adolescente que cursa E.S.O. en Irun. Pero también pretende beneficiar a los actores que intervienen en el fomento de la actividad física en este grupo de población, de manera que mejoren sus formas de hacer, que adquieran conocimientos sobre la intervención con los jóvenes y puedan resultar más efectivos en su acción.

¿Para qué? ¿qué? y ¿cómo? Qué queremos conseguir, qué vamos a hacer y cómo lo vamos a hacer constituyen los contenidos de otro apartado. Aquí se han diferenciado claramente dos áreas. Por un lado, el desarrollo de un Marco para la Acción y por otro lado el Plan de Actuación. El Marco para la Acción sienta las bases y la orientación filosófica del programa, tratando de ofrecer una guía para la acción sustentada en el mejor conocimiento disponible.

El Plan de Actuación incluye los procesos habilitados para el diseño y ejecución de intervenciones que permitan alcanzar los objetivos planteados. Las percepciones de las personas (análisis cualitativo a partir de grupos focales), los determinantes de la actividad física y la conducta sedentaria y la evidencia sobre estrategias que pueden ser efectivas

PROGRAMA IKAS-SASOI - FASES DEL PROYECTO

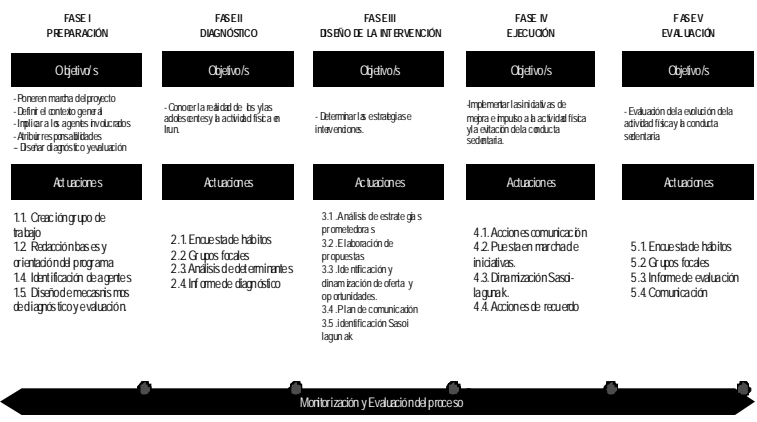

Figura 3. Fases y contenidos del programa IkasS asoi. Fuente: elaboración pro pia basado en Ituríoz et al., 2011. 
constituyen las tres fuentes sobre las que construir las propuestas de intervención.

Es previsible, a la vista de la literatura científica disponible, que los resultados conduzcan a largo plazo a la consecución de unos beneficios traducidos en mejora de indicadores de salud, mejora de indicadores medioambientales, ahorro en el gasto sanitario, empleo en el sector de la actividad física y otros.

Participación y evaluación están presentes a lo largo de todo el desenvolvimiento del proceso.

Las labores a llevar a cabo con este esquema metodológico de referencia se han distribuido en cinco fases: lanzamiento, bases del plan, propuestas de intervención, preparación para la intervención y puesta en marcha. En la Figura 3 se refleja la descripción de las fases en la gestión de la iniciativa.

\section{Participantes}

IkasSasoi está dirigido a los y las adolescentes que cursan estudios de E.S.O. en los centros de Irun participantes en el programa. Se han definido como actores involucrados en el programa:

- Alumnos y alumnas de E.S.O. de Irun

- Ayuntamiento de Irun (Servicio de Deportes, Juventud, Intervención Comunitaria, Igualdad, Educación y Movilidad)

- Centros Educativos (los siete centros de E.S.O. existentes en el municipio: I.E.S. La Salle, I.E.S. El Pilar, I.E.S. Txingudi Ikastola, I.E.S. San Vicente de Paul, I.E.S. Toki Alai, I.E.S. Hirubide, PCPI Bidasoa)

- Otros: Dirección Territorial de Salud Pública de Gipuzkoa, centros de salud de Irun, Grupo de Actividad Física y Salud de OsatzenSociedad Vasca de Medicina de Familia y Comunitaria, empresa gestora Gazteleku (centro de jóvenes), empresa gestora Programa Medio Abierto de Irun (educadores de calle), clubes deportivos y gimnasios y empresas de servicios deportivos.

\section{Diagnóstico}

Con el fin de obtener información relevante se administró en las aulas de los institutos un cuestionario de hábitos de actividad física y otros hábitos saludables a un total de 1.797 alumnos y alumnas de Primero a Cuarto curso de la ESO. Los datos preliminares indican que un $14 \%$ de los chicos y un $25 \%$ de las chicas no alcanzan una actividad mínima en el tiempo libre de 150 minutos semanales de actividad física moderada o vigorosa (AFMV). Sólo un 3,4\% de las personas encuestadas se sitúa en un estadio precontemplativo: no es muy activo, ni tiene intención de hacer nada de actividad física, lo cual procura un amplio margen para la labor de fomento. Un 12,5\% refiere ubicarse en un estadio de abandono. Menos del 8\% de los encuestados considera que no es fácil acceder a actividades o espacios para hacer actividad física en la ciudad. Un 47,6\% no encuentra barrera alguna para realizar actividad física, registrándose como principales barreras: no disponer de tiempo, que no les guste practicarlo solas, no les gusta que les vean. Un $51 \%$ de los chicos y un 58\% de las chicas de estas edades pasan más de dos horas frente a pantallas en días laborables. Esta cifra alcanza el 77\% y el $79 \%$ respectivamente los fines de semana.

Con el fin de ampliar el espectro de respuestas obtenidas a través de las encuestas y profundizar en aspectos significativos para la intervención se llevaron a cabo cuatro grupos focales, tres grupos de alumnos y alumnas de ocho participantes cada uno y un grupo focal compuesto por el personal docente de los centros. Fruto de la información obtenida por estas vías, se recogieron una serie de planteamientos sobre los que elaborar el plan de acción a desarrollar durante los cursos 2013-14 y 2014-15. Las propuestas se agruparon, tal como se relaciona a continuación, en cuatro bloques para su mejor comprensión, diferenciando las acciones que no inciden directamente en el ámbito del centro educativo (Acciones comunitarias), las que inciden en el centro escolar (Centro educativo), acciones informativas o de marketing (Comunicación)y las que se ligan directamente al transporte activo (Movilidad).

Acciones comunitarias

- Oferta integrada de actividades en Irun.
· Eliminar lanorma de límite de acceso a gimnasios de polideportivos a menores de 16 años.

- Identificar espacios en Irun para la práctica de actividad física, accesibles para los jóvenes.

- Generar y habilitar espacios de libre acceso para hacer actividad física, en los barrios y en el centro.

- Salidas a la naturaleza los fines de semana.

Facilitar prueba de las actividades que ya existen.

- Instalar wifi gratuito en zonas deportivas como forma de atracción.

Competiciones entre locales

- Semana activa en los centros de salud. Consejo de actividad física en consulta de pediatría (12-16 años).

Campaña descuento en material deportivo a jóvenes de Secundaria.

- Oferta servicios a medida.

Facilitar información y acceso a ferias del deporte.

Centro educativo.

- Utilizar las sesiones de Educación Física para promover deportes distintos, acudir a instalaciones, probar actividades.

- Que el profesor de Educación Física disponga de información sobre todas las actividades que se pueden realizar.

- Promover actividades conjuntas entre centros.

- Abrir patios de los colegios fuera del horario escolar.

- Espacios para el uso del scooter, skate... en los patios de los centros.

Campeonatos para alumnos en los recreos.

- Préstamo de material deportivo para los recreos.

- Sasoi-laguna. Alumno referente que ayuda a los demás a ser activos.

- Informar a los centros sobre las ofertas de Diputación para hacer actividades del programa de deporte escolar. Incidir sobre precios y uso del idioma.

Comunicación

- Información sobre equipos de deportes.

Comunicación periódica a los padres: recomendaciones de actividad física, conductas sedentarias, deporte y resultados académicos..

Acciones de comunicación

- Acciones Internet.

- Boletín Gazte Sasoia (txikis del Bidasoa).

Movilidad

- No multar a los chicos y chicas que se desplazan sobre ruedas en las aceras.

- Canalizar y fomentar el uso de bidegorri para acudir a la playa.

- Custodia de bicicletas, scooters... en los colegios.

Empleo de podómetro como incentivador de la actividad física.

\section{Plan de Acción}

El grupo de trabajo interdisciplinar de IkasSasoi aprobó llevar a cabo a partir de las propuestas anteriores un total de 23 intervenciones a desarrollar durante el curso 2013-14 y 2014-15. De entre ellas, seis acciones implican la elaboración de un estudio para analizar su viabilidad de manera previa a su ejecución. Se exponen a continuación las acciones aprobadas por el grupo:

i.1. Comunicación de la oferta de actividades de Irun a comienzo de curso.

i.2. Comunicación de catálogo de espacios para la práctica de actividades físico-deportivas de Irun.

i.3. Eliminar la norma de límite de acceso a gimnasios de polideportivos a menores de 16 años.

i.4. Mes IkasSasoi, en el que se hará especial énfasis en los centros de salud en el consejo de actividad física a este grupo de población. i.5. Estudio: apertura de las instalaciones deportivas de determinados centros educativos a la comunidad en horario extralectivo. i.6. Campaña descuento en material deportivo a jóvenes de Secundaria. 
i.7. Comunicación a centros de E.S.O. de Irun sobre oferta de actividades de promoción incluidas en la campaña de deporte escolar de Diputación.

i.8. Comunicación a centros de E.S.O. de Irun sobre catálogo de actividades a realizar en horario lectivo de educación física en Irun. i.9. Apertura de espacio «IkasSasoi» en la web irunkirol.

i.10. Instalar aparcamientos seguros para bicicletas y scoots/ longboards/skates en los centros educativos de E.S.O.

i.11. Formación a jóvenes sobre pautas y normas para desplazarse activamente en la ciudad.

i.12. Estudio: Organizar salidas intercentros de E.S.O. a la naturaleza.

i.13. Préstamo de material deportivo en los recreos en los centros educativos de E.S.O.

i.14. Acceso a ferias y eventos deportivos

i.15. Estudio: instalar wi-fi gratuito en zonas deportivas como forma de atracción.

i.16. Estudio: oferta de actividades interlocales.

i.17. Servicio de préstamo de podómetros a alumnos/as de E.S.O.

i.18. Habilitación de espacios urbanos para la práctica de actividad física de los y las jóvenes.

i.19. Estudio: Sasoi-laguna, referentes que ayudan a compañeros/as a ser activos/as.

i.20. Boletín IkasSasoi para padres y madres.

i.21. Presentación del programa IkasSasoi.

i.22. Foro intercentros IkasSasoi.

i.23. Estudio: Teléfono móvil+ IkasSasoi.

A cada una de estas intervenciones le ha sido asignado un responsable, un cronograma, una delimitación de acciones a llevar a cabo e indicadores de evaluación. Esta información para cada acción queda recogida en un modelo de ficha. A modo de ejemplo, en la Figura 4 se presenta la ficha correspondiente a la acción «i.1, comunicación de la oferta de actividades de Irun a comienzo de curso».

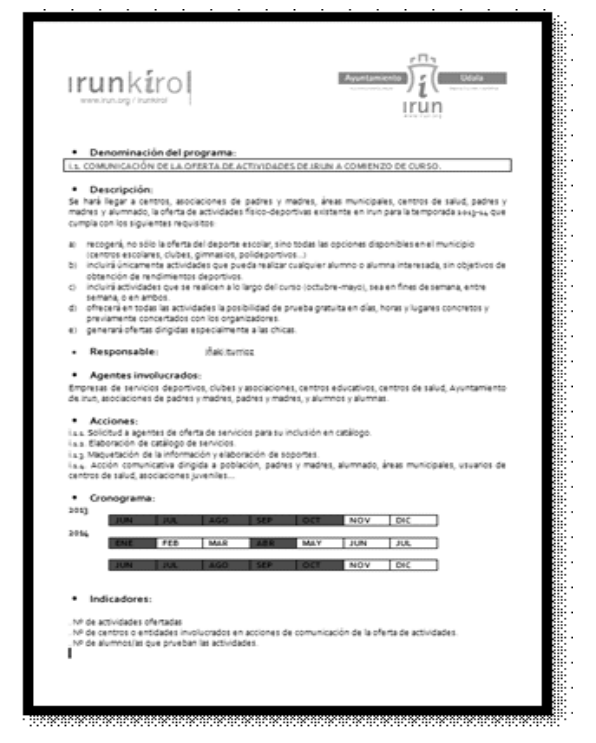

Figura 4. Ficha de control relativa a la acción i.1. Comunicación de la oferta de actividades de Irun a co mienzo de curso.

\section{Resultados}

Se ha desarrollado una monitorización sobre el desarrollo del proyecto a modo de evaluación de proceso. Hasta el momento se han llevado a cabo en el curso tres reuniones de seguimiento en fechas del 23 de octubre y 12 de marzo de 2013, y 26 de noviembre de 2014. En esta última se ha verificado la ejecución o puesta en marcha de 19 de las 23 intervenciones aprobadas. El grupo percibe mejoras sensibles en la coordinación de agentes para actuar en el proyecto, el desarrollo de las iniciativas, los cambios que se están introduciendo en las políticas y una mayor atención por parte de agentes de la comunidad a este grupo de población. El programa ha generado también la puesta en marcha de otros proyectos colaborativos a través de la red creada por el programa IkasSasoi.

La evaluación de resultados se realiza sobre cada una de las acciones de intervención en función de los indicadores que tienen asignados. Se muestran a continuación los productos y resultados de acciones llevadas a cabo en el marco de la campaña.

i.1. Comunicación de la oferta de actividades de Irun a comienzo de curso: 18 actividades recreativas (no competitivas), 17 de ellas con posibilidad de prueba gratuita en el curso 2013/14. Un total de 23 actividades ofertadas en el curso 2014/15. 17 empresas y clubes de la ciudad ofertando actividades. Mejoras indicadas por las empresas y asociaciones que han participado en la oferta de actividades: incremento en 23 inscripciones a hípica, nuevo grupo de atletismo.

i.2. Comunicación de catálogo de espacios para la práctica de actividades físico-deportivas de Irun: cuatro acciones de comunicación, denominadas Planes Irunkirol. Se distribuye información sobre instalaciones en Irun para la práctica de pelota, baloncesto, fútbol y nueva zona recreativa de juego. Se indica emplazamiento y fórmula de acceso.

i.3. Eliminar la norma de límite de acceso a gimnasios de polideportivos a menores de 16 años. En el período del trimestre octubre-diciembre de 2013, momento inicial de la implantación de la nueva norma, se han registrado un saldo neto de 36 nuevas altas correspondientes a este tramo de edad. La norma se mantiene en vigor. Además se han incorporado otras medidas:

Descuento de matrícula a jóvenes entre 13 y 22 años para el período julio-septiembre. En este período se registraron 70 altas de personas de este grupo de edad, el $10 \%$ del total de las altas. El peso parcial que representa ordinariamente este grupo de población sobre el total de abonados es del $4 \%$, por lo que se estima muy favorablemente el impacto de la medida.

Campaña de promoción de abonados/as 2014-15: desde comienzos de septiembre se han registrado 26 altas de personas entre 13 y 22 años. Esto supone un $8 \%$ del total de altas. Duplica también el peso relativo ordinario de total de abonados/as.

Consultados profesionales de los polideportivos, refieren observar más usuarios/as en las salas de fitness.

i.4. Mes IkasSasoi en los centros de salud: el Ayuntamiento está colaborando con Osakidetza en el Plan de Prevención de la Enfermedad Cardiovascular de la comarca Bidasoaldea, una iniciativa comunitaria para intervenir en los factores de riesgo que incluye la inactividad física y otros hábitos. Es previsible el desarrollo de una iniciativa de estas características o similar en el marco del Plan. No se ha desarrollado hasta el momento.

i.5. Estudio: apertura de las instalaciones deportivas de determinados centros educativos a la comunidad en horario extralectivo: se ha puesto en marcha el programa Patioetan Sasoi. Desde octubre de 2014 se abren en horario extraescolar los patios de tres centros: Belaskoenea, Dunboa y Eguzkitza, y en 2015 se va a incorporar Leka Enea. Ayuntamiento y centros educativos se han coordinado para la ejecución del programa. Total de horas adicionales de apertura de las instalaciones de los patios: $5.373 \mathrm{~h}$ durante 215 jornadas al año.

i.6. Campaña descuento en material deportivo a jóvenes de Secundaria: se tramitó la propuesta en colaboración con el Área de Promoción económica. La época pareció inadecuada por coincidir con época de ventas. Algún comercio ha mostrado interés posteriormente, pero de manera inconsistente.

i.7. Comunicación a centros de E.S.O. de Irun sobre oferta de actividades de promoción incluidas en la campaña de deporte escolar de Diputación: formulada la solicitud, un representante de la Diputación Foral de Gipuzkoa acudió a explicar el contenido y funcionamiento a los coordinadores de los centros educativos de Primaria y Secundaria.

i.8. Comunicación a centros de E.S.O. de Irun sobre catálogo de actividades deportivas a realizar en horario lectivo de Educación Física en colaboración u ofrecidas por asociaciones yempresas deportivas de Irun: si bien no se ha elaborado un catálogo general, se han encaminado acciones de promoción dentro del horario lectivo en modalidades 
como rugby y tenis de mesa.

i.9. Apertura de espacio «IkasSasoi» en la web irunkirol: la web irun.org/irunkirol cuenta con un banner en el que se va colgando la información del programa.

i.10. Instalar aparcamientos seguros para bicicletas y scoots/ longboards/skates en los centros educativos de E.S.O.: se ha iniciado una colaboración con otras áreas municipales, pero no se han iniciado las intervenciones.

i.11. Formación a jóvenes sobre pautas y normas para desplazarse activamente en la ciudad: Alo largo de 2013-2014 se han desarrollado sesiones de conducción de bicicletas en medio urbano para alumnos de dos centros de E.S.O. de Irun. Para ello se han captado recursos de programas de Gobierno Vasco. Participación de 90 alumnos (cuatro aulas) de dos centros educativos.

i.12. Estudio: Organizar salidas intercentros de E.S.O. a la naturaleza: se han ofertado actividades a través de la asociación Txiribuelta. También se han organizado salidas dentro del programa Nordic Sasoi (iniciación a la modalidad de nordic walking en el entorno natural de Irun). No obstante, estas actividades, que fueron propuestas por los y las jóvenes con el formato planteado, han tenido menor repercusión de la esperada. Se organizaron dos salidas gratuitas con Txiribuelta y cinco dentro del programa Nordic sasoi, en estas últimas participaron un tal de 16 jóvenes.

i.13. Préstamo de material deportivo en los recreos en los centros educativos de E.S.O.: se está tramitando la contratación del suministro de cuatro carros de material deportivo alternativo que se facilitarán a los centros de E.S.O. de manera rotatoria. En enero de 2015 se acordará con los centros la manera de desarrollar la iniciativa. El material podrá estar también a disposición de los educadores de calle. Importe de la inversión en material deportivo: $4.999,00$ •

i.14. Acceso a ferias y eventos deportivos: a lo largo de los dos cursos se han distribuido 65 entradas a adolescentes a través de centros de E.S.O. y del programa de Medio Abierto.

i.15. Estudio: instalar wi-fi gratuito en zonas deportivas utilizando el atractivo de disponibilidad de wifi como forma de atracción a espacios para el deporte en lugar de espacios favorecedores de la conducta sedentaria: el Ayuntamiento ha puesto en marcha el acceso a wi-fi gratuito durante un máximo de dos horas diarias a través de la Iruntxartela. La cobertura alcanza a buena parte del parque de instalaciones deportivas municipales.

i.16. Estudio: oferta de actividades interlocales a los y las jóvenes que alquilan locales como lugar de encuentro con amigos y amigas en el tiempo de ocio: se está elaborando un programa desde el Servicio de Juventud del Ayuntamiento que contempla actividades físicas en el medio urbano.

i.17. Servicio de préstamo de podómetros a alumnos/as de E.S.O.: se distribuyeron a lo largo del curso 2013-14 dos podómetros a cada uno de los centros educativos con las bases del programa para tratar de incentivar la actividad física especialmente en las personas más inactivas. Se ha recabado bajo nivel de impacto con la acción, se refieren únicamente 5 participaciones por parte de un centro. Se llevará a cabo un recordatorio en el mes de enero de 2015.

i.18. Habilitación de espacios urbanos para la práctica de actividad física de los y las jóvenes: se va a construir una instalación de barrio en Urdanibia Berri. Se ha contemplado en el diseño su utilización por el grupo de población joven. Se ha introducido en el presupuesto 2015 una propuesta de inversión en material deportivo. Una de las prioridades, atendiendo a demandas realizadas directamente por jóvenes, es la de crear un gimnasio al aire libre. Este gimnasio podrá servir de apoyo a los grupos de parkour de Irun.

i.19. Estudio: Sasoi-laguna, referentes que ayudan a compañeros y compañeras a ser personas activas: esta iniciativa no se ha iniciado por el momento. Se trata de captar a jóvenes líderes que actúen como intermediarios entre el grupo de trabajo del programa y los jóvenes en los centros educativos.

i.20. Boletín IkasSasoi para padres y madres: se realizó una intervención en el curso 2013-14 sobre la importancia del proceso, de la mejora versus el resultado en el deporte. A lo largo del curso 2014-15 están previstas tres acciones.

i.21. Presentación del programa IkasSasoi: el programa fue presentado a través de rueda de prensa y acción comunicativa municipal el 27 de septiembre de 2013. En septiembre de 2014 se ha llevado a cabo una nueva acción comunicativa.

i.22. Foro intercentros IkasSasoi: se han producido intercambios de impresiones y experiencias en el marco de las reuniones del grupo de trabajo. En el caso de centros que no han podido acudir a las reuniones se han mantenido contactos individuales desde Irunkirol.

i.23. Estudio: Teléfono móvil+ IkasSasoi: se trata de estudiar abrir una vía de comunicación directa con los y las jóvenes a través de la aplicación whatsapp para temas vinculados al programa IkasSasoi. Se ha analizado la propuesta, concluyéndose una necesidad de mejor coordinación con la Oficina de Información Juvenil para utilizar sus canales de información, dado que disponen de canales apropiados de comunicación con este grupo de población.

\section{Conclusiones}

El grupo de trabajo interdisciplinar para el seguimiento del programa considera que el programa IkasSasoi ha permitido generar determinados impactos positivos en relación con los objetivos del programa. En primer lugar, ha permitido conocer en profundidad los hábitos y percepciones de las y los jóvenes en relación con la actividad física. Además, ha generado una red intersectorial (grupo de trabajo y otros agentes de la comunidad) consolidada, lo que ha dado cauce a un mejor conocimiento de los agentes que intervienen en la promoción de actividad física dirigida a este colectivo y ha facilitado la coordinación de otras iniciativas y programas. Se valora positivamente también, a nivel operativo, haber dado cauce a la implementación de diecinueve acciones de intervención en este ámbito. En definitiva, el programa está consiguiendo activar a la comunidad en apoyo a la actividad física de los y las jóvenes y ha ubicado a los jóvenes de estas edades en la agenda de las políticas locales.

Desde la perspectiva del personal docente de Educación Física, intervenciones multicomponente de acción comunitaria pueden suponer un valioso apoyo en la labor de creación de hábitos de práctica de actividad física regular en la población. Puede contribuir también a mejorar la percepción del valor de la Educación Física en la educación integral de las personas y conducir así a la adquisición de una mayor relevancia dentro de la comunidad escolar.

Los principales aspectos a mejorar detectados se refieren al incumplimiento de los plazos de intervención en acciones concretas y a la desigual implicación de los agentes, especialmente en los centros educativos. En concreto, en determinados centros, se han encontrado dificultades en los interlocutores para disponer de tiempo para asistir a las reuniones, lo que ha exigido al personal del Ayuntamiento de Irun una mayor dedicación y atención para lograr que las intervenciones alcancen al alumnado de estos centros. Esta limitación de recursos humanos se ha debido fundamentalmente a las dificultades para cubrir las ausencias de los docentes de los departamentos de Educación Física o al desempeño de tareas directivas adicionales por parte de este personal. Si bien en la mayoría de los centros se ha mantenido un cauce de comunicación fluido y se ha encontrado un importante apoyo al programa, cabría estudiar la posibilidad de establecer el vínculo con los centros escolares a través de los tutores y tutoras, tal y como se ha realizado en experiencias de éxito en el entorno próximo. No obstante, debe tenerse en cuenta que esto requeriría una extensión en el número de interlocutores con el que coordinarse.

El programa cuenta con continuidad en la mayoría de las acciones que se han estimado viables. El Ayuntamiento, en colaboración con los agentes del programa, ha fijado realizar cada cuatro años un seguimiento de los hábitos de actividad física en este grupo de población. En primavera del curso 2014/15 se realizará un nuevo seguimiento a través de cuestionario para valorar inicialmente la evolución de los hábitos a partir de la puesta en marcha de las medidas adoptadas. Se pretende también 
encontrar aliados para realizar una medición objetiva complementaria de los niveles de actividad física, hasta el momento no ha resultado fructífera esta búsqueda de investigadores que utilicen acelerometría en el entorno próximo.

IkasSasoi es un programa incluido en el programa comunitario Irun Sasoian que persigue generar condiciones favorables en la ciudad para el fomento de la actividad física. Existen otras iniciativas dentro del programa Irun Sasoian que inciden en el grupo de población adolescente en cuanto a impulso de un urbanismo favorecedor de la actividad física, movilidad activa y creación de oportunidades para la práctica de actividad física. Puede citarse, a modo de ejemplo, la edición de un código de Urbanismo Activo para la ciudad (Urban Sasoi), los programas de dinamización del espacio urbano para la actividad física (Igandesasoi y ArtSasoi), la creación de foros para la discusión de políticas de fomento de la actividad física (Foro Profesional Irun Sasoian) o la colaboración en los programas «camino escolar».

\section{Referencias}

Abarca-Sos, A., Zaragoza, J., Generelo, E., \& Julián, J. A. (2010). Comportamientos sedentarios y patrones de actividad física en adolescentes. Revista Internacional de Medicina y Ciencias de la Actividad Física y del Deporte, 40 (39), 410-427.

Bellew B., Bauman A., Martin B., Bull F., \& Matsudo V. (2011). Public policy actions needed to promote physical activity. Curr. Cardiovasc. Risk Rep., 5,340-349.

Biddle S., \& Asare M. (2011). Physical activity and mental health in children and adolescents: a review of reviews. Br J Sports Med 2011, 45, 886-895. doi:10.1136/bjsports-2011-090185

Brown W.J., Bauman A.E., \& Owen N. (2009). Stand up, sit down, keep moving: turning circles in physical activity research? $\mathrm{Br} . J$. Sports. Med., 43, 86-88.

Consejo Superior de Deportes (2011). Estudio los hábitos deportivos de la población escolar en España. Madrid. Consejo Superior de Deportes.

Crutzen R. (2010). Adding effect sizes to a systematic review on interventions for promoting physical activity among European teenagers. International Journal of Behavioral Nutrition and Physical Activity. 7:29. doi:10.1186/1479-5868-7-29

Currie C. et al. (2008). Inequalities in young people's health: HBSC international report from the 2005/06 Survey. Health Policy for Children and Adolescents, No. 5. Copenhagen. WHO Regional Office for Europe.

Dobbins M., Husson H., DeCorby K., \& LaRocca R.L. (2013). Schoolbased physical activity programs for promoting physical activity and fitness in children and adolescents aged 6 to 18.Cochrane Database of Systematic Reviews. Issue 2, Art. No.: CD007651.

Excmo. Ayuntamiento de Irun. Acuerdo plenario de 28 de marzo de 2012.

Fedewa, A. L., \& Ahn, S. (2011). The effects of physical activity and physical fitness on children's cognitive outcomes: A meta-analysis. Research Quarterly for Exercise and Sport, 82(3), 521-535.

Global Advocacy for Physical Activity (GAPA) y Advocacy Council of the International Society for Physical Activity and Health (ISPAH). (2011). NCD Prevention: Investments that Work for Physical Activity. Recuperado de: htpp://www.globalpa.org.uk/ investmentsthatwork.

Hamilton M.T., Hamilton D.G., \& Zderic T.W. (2007). Role of low energy expenditure and sitting in obesity, metabolic syndrome, type 2 diabetes, and cardiovascular disease. Diabetes, nov. 56(11), 2655-67.

Hernández L.A., Ferrando J.A., Quilez J., Aragonés M., y Terreros J.L.(2010). Análisis de la actividad física en escolares del medio urbano. Investigación en Ciencias del Deporte. 55. Madrid. Consejo Superior de Deportes.

Huhman M., Heitzler C., \& Wong F. (2004). The Verb campaign logic model: a tool for planning and evaluation. Prev Chronic Dis., 1, A11.

Iturrioz I. et al. (2011). Aktibili. Marco para la Acción. Vitoria-Gasteiz. Gobierno Vasco. Recuperado de: https://www6.euskadi.net/r46- keeduk/eu/contenidos/informacion/kiroleskola/eu_kirolesk/adjuntos/Aktibili_marco_para_la_accion_version_0.1.pdf

Iturrioz, I. (2012a). De Servicio municipal a Patronato y de Patronato a Servicio municipal: el caso de Irun. Lecciones aprendidas. Diputación Foral de Bizkaia. No publicado. Recuperado de: http:// www.bizkaia.net/home2/archivos/DPTO4/Temas/

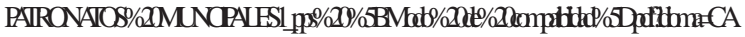

Iturrioz I. (2012b). Modelo vasco de actividad física. Un marco referencial para la promoción de la actividad física en Euskadi. En: Martínez de Aldama I., Ortúzar I., Cayero R., y Calleja J. (Coord) et al.: Investigación e innovación en el deporte. (pp. 352-358). Barcelona. Paidotribo.

Iturrioz I. (2012c). Recomendaciones de actividad física saludable dirigidas a la población vasca. En: Martínez de Aldama I., Ortúzar I., Cayero R., y Calleja J. (Coord) et al.: Investigación e innovación en el deporte. (pp. 384-396). Barcelona. Paidotribo.

Janssen I, \& Leblanc A. (2010). Systematic Review of the Health Benefits of Physical Activity in School-Aged Children and Youth. International Journal of Behavioral Nutrition and Physical Activity 7:40.

Kelly P., Matthews A. \& Foster Ch. (2012). Young and physically active: a blueprint for making physical activity appealing to youth. Génova. Organización Mundial de la Salud. Recuperado de: http:/ /www.euro.who.int/_data/assets/pdf_file/0005/175325/ e96697.pdf

Kriemler S., Meyer U, Martin E., van Sluijs E.M.F., Andersen L.B., \& Martin B.W. (2011). Effect of school-based interventions on physical activity and fitness in children and adolescents: a review of reviews and systematic update. Br J Sports Med. 45:11 923930.

Mulholland E. (2008). What Sport can do. The True Sport Report. Ottawa. Canadian Centre for Ethics in Sport. Recuperado de: http:/ /www.cces.ca/files/pdfs/TS_report_EN_webdownload.pdf

Murillo B., García E., Generelo E., Bush P.L., Zaragoza J., Julián J.A., y García González L. (2013). Promising school-based strategies and intervention guidelines to increase physical activity of adolescents. Health Educ Res. Jun, 28(3), 523-38.

Organización Mundial de la Salud (2010). Recomendaciones mundiales sobre actividad física para la salud. Génova. Organización Mundial de la Salud. Recuperado de: http://whqlibdoc.who.int/publications/ 2010/9789243599977_spa.pdf

Oviedo G. et al., (2013). Niveles de actividad física en población adolescente: estudio de caso. Retos. Nuevas tendencias en Educación Física, Deporte y Recreación, $n^{\circ}$. 23, 43-47.

Singh A., Uijtdewilligen l., Twisk J., van Mechelen W, \& Chinapaw M., (2012). A systematic review of the literature including a methodological quality assessment. Arch Pediatr Adolesc Med., 166(1), 49-55.

Telama R., Yang X., Viikari J., Wanne O., \& Raitakari O. (2005). Physical activity from childhood to adulthood: a 21-year tracking study. Am J Prev Med., Apr., 28(3), 267-73.

Van Sluijs E. M., McMinn A. M., \& Griffin S. J., (2007) Effectiveness of interventions to promote physical activity in children and adolescents: systematic review of controlled trials. B.M.J. Oct 6, 335:703.

US Department of Health and Human Services (2002). Physical Activity Evaluation Handbook. Atlanta. US Department of Health and Human Services, Centers for Disease Control and Prevention.

Villasante, T. (2010). «Historias y enfoques de una articulación metodológica participativa», Cuadernos CIMAS-Observatorio Internacional de Ciudadanía y Medio Ambiente Sostenible. 1- 18. Recuperado de: http://www.redcimas.org/archivos/biblioteca/ metodologias/TVillasante_HISTORIAS.pdf

World Health Organization (2007). A guide for population-based approaches to increasing levels of physical activity: implementation of the WHO global strategy on diet, physical activity and health. Geneve. World Health Organization.

World Health Organisation (2009). Interventions on Diet and Physcial Activity: What Works? Summary Report. Geneve. World Health Organization. 